\title{
Comparative Effects of Improved Cardiorespiratory Fitness, Stress, and Weight on Blood Pressure in a Community-Based Treatment: Implications for Physician Referral
}

\author{
James J. Annesi* \\ YMCA of Metropolitan Atlanta, 100 Edgewood Avenue, NE, Suite 1100, Atlanta, GA, USA
}

\begin{abstract}
Introduction: The efficacy of behavioral treatments for elevated blood pressure (BP) is unclear, and the relative effectiveness of improvements in common intervention targets such as cardiorespiratory fitness, stress, and body weight requires clarification. Aims of this research were to assess the effectiveness of a community-based, health behaviorchange program for improving resting heart rate, weight, and stress; and to clarify associations of their changes with BP changes. Methods: A group of 140 women with severe obesity volunteered for a YMCA-based treatment for weight loss incorporating exercise, nutrition, and stress management methods. Physiological and psychological measurements were obtained at baseline and month 6. Results: The treatment was associated with significant within-group improvements in resting heart rate, tension, weight, and systolic and diastolic BP (all $P$ values $<0.01$ ). The moderate effect sizes for BP change ( $d=0.37$ and 0.58 , respectively) were comparable with more invasive and expensive behavioral treatments. Significant linear bivariate relationships were found between changes in resting heart rate, tension (stress), and weight, and systolic and diastolic BP changes. When simultaneously entered into multiple regression equations, the variance accounted for in systolic and diastolic BP $\left(R^{2}=0.17\right.$ and 0.06 , respectively) was significant. In each equation, only changes in tension significantly contributed independently to the explained variances in BP changes. Conclusions: Along with improvements in fitness and weight, managing stress appears to be especially important for reducing BP. The present community-based treatment may be a viable option for physician referral as either an alternate or adjunct to medications.
\end{abstract}

Keywords: Blood pressure, hypertension, cardiovascular fitness, stress, weight change.

\section{INTRODUCTION}

As of the most recent analyses, hypertension affected $31 \%$ of adults or 65 million people in the United States, making control of elevated blood pressure (BP) one of the most frequent activities of medical professionals [1]. Although there is clear evidence of the usefulness of antihypertension medications for controlling high BP [2], there is also a realization that long-term drug treatment is expensive, side effects may be considerable, and patients may wish to avoid long-term drug use when possible. Growing interest in non-pharmacological treatments for hypertension led to the development of 2 expert panels to consider behavioral interventions [3,4]. Both panels cited methodological limitations in the extant research and stated that considerable investigation remains necessary prior to determining both the efficacy and the most productive foci of behavioral treatments [3,4]. Notably, however, in a comprehensive "review of reviews" of behavioral BP treatments, some methods demonstrated effects comparable to medications, with the average of all 28 effect sizes of preto post-treatment changes in $\mathrm{mmHg}$ in the moderate range of $d=-0.44$ and $d=-0.46$ for systolic and diastolic BP, respectively [5]. Initial BP was negatively related to BP improvements [5].

*Address correspondence to this author at the YMCA of Metropolitan Atlanta, 100 Edgewood Avenue, NE, Suite 1100, Atlanta, GA, USA;

Tel: 404.267.5355; Fax: 404.527.7693; E-mail: JamesA@ymcaatlanta.org
Common aims of the behavioral treatment of elevated BP is to improve individuals' cardiorespiratory fitness, reduce stress (manifested as anxiety or psychological tension), and reduce excess weight. The association of improvement in cardiorespiratory fitness and decreased BP is strong [6], with resting heart rate often used as a marker of fitness [7]. Mechanisms causing these effects appear to be related to the association between a strengthened heart and the body's ability to then pump more blood with less effort, thus decreasing the force on the arteries [8]. The rationale for reducing stress to improve $\mathrm{BP}$ is through the link that stress is thought to have with hypertension via elevated sympathetic tone and vagal dysregulation [9]. Training in various relaxation methods (e.g., biofeedback, progressive relaxation, yoga) emphasizes reductions in physiological arousal and improved autonomic balance. Research has demonstrated the association of reduced stress and improved BP [10]. The reasoning for reducing one's body weight to improve $\mathrm{BP}$ is less evident, although research clearly demonstrates the association [11]. One plausible pathway is that the renin-angiotensin-aldosterone system is overactivated in obese individuals, and aldostrone concentrations are higher than in persons of a normal weight [12]. Decreased insulin sensitivity and hyperinsulinemia as part of the metabolic syndrome might also form a link [13]. Little research has tested the relative effectiveness of improving cardiorespiratory fitness, tension, or weight, although such data may help to shape the effectiveness and efficiency of behavioral treatments (e.g., by prioritizing corresponding intervention 
components such as exercise, stress management techniques, or nutrition change). It is also unknown if meaningful effects may be obtained by referral to cost-effective communitybased programs, or whether more expensive processes (e.g., individual meetings with a licensed psychologist) are necessary.

Thus, the present research tested a group of severely obese women enrolled in a 6-month YMCA-based treatment focused on weight management that included components of exercise, nutrition, and stress-management. Although each participant had an initial BP value consistent with either prehypertension or hypertension, the stated focus of the treatment was weight-loss, so expectations for BP improvement were not likely to bias outcomes, as has been cited as problematic in earlier research [10]. It was hypothesized that the treatment, based on tenets of social cognitive and selfefficacy theory (which views individuals as directing their own behaviors based on self-reflection, self-organization, and perceptions of ability) [14,15], would be associated with significantly improved resting heart rate, tension, weight, and BP. Changes in resting heart rate, tension, and weight were each expected to be significantly related to both systolic and diastolic BP improvements. Which predictor of BP change would be strongest was, however, left as a research question without hypotheses. It was hoped that findings would serve to inform behavioral treatments for high BP. Ultimately, physicians and other medical professionals might benefit by allowing them to better select efficient and cost-effective referrals for their patients to potentially serve as both alternatives and adjuncts to antihypertension drugs.

\section{METHODS}

\section{Participants}

Women responded to advertisements in local newspapers soliciting volunteers for a YMCA-based weight-loss program. Inclusion criteria were: 1) minimum age of 21 years, 2) BMI of $35-50 \mathrm{~kg} / \mathrm{m}^{2}, 3$ ) no regular exercise (less than 20 minutes per week average), 4) a goal of weight loss, and 5) systolic and/or diastolic BP at or above the recommended value of 120 or $80 \mathrm{mmHg}$, respectively. Exclusion criteria were current or soon-planned pregnancy, use of medications for weight loss or a psychiatric condition, and, if medications for BP were presently being used, they could not have been initiated or changed either within the previous 6 months or during the investigation. A written statement of adequate physical health to participate from a physician, appropriate institutional review board approval, and written informed consent from all study participants was obtained.

Of the 88 participants classified as pre-hypertensive (systolic BP of 120-139 mmHg and/or diastolic BP of 80-89 $\mathrm{mmHg}$ ) and 52 classified as hypertensive (systolic BP $\geq 140$ $\mathrm{mmHg}$ and/or diastolic $\mathrm{BP} \geq 90 \mathrm{mmHg}$ ), the overall mean age was 45.1 years $(\mathrm{SD}=9.8)$. The mean body mass index (BMI) was $40.4 \mathrm{~kg} / \mathrm{m}^{2}(\mathrm{SD}=4.4)$. The overall racial makeup was $43 \%$ white, $51 \%$ black, and $6 \%$ of other races/ ethnicities. Based on self-reported data indicating family income, most were in the lower-middle to middle class.

\section{Measures}

Cardiorespiratory fitness was measured as resting heart rate. This was assessed as beats per minute after a minimum of 5 minutes rest. A recently calibrated digital scale was used to measure weight in $\mathrm{kg}$. An aneroid sphygmomanometer with stethoscope $\left(\mathrm{Omron}_{\circledast}\right.$ Exactus $108 \mathrm{MC}$ for Large Adults, South Shelton, CT, USA) was used to measure systolic and diastolic BP in mmHg. Measurements were each taken at a similar time of day by the same technician and the average of 2 measurements was recorded.

Psychological tension was measured by the Profile of Mood States Short Form [16]. The 5-item tension scale (e.g., nervous, anxious, tense) required responses ranging from 0 (not at all) to 4 (extremely) to indicate "how you have been feeling during the past week including today." The internal consistency was 0.91 , and test-retest reliability over 3 weeks was $0.70[16]$.

\section{Procedure}

Each participant was provided full access to a YMCA facility and received an orientation to study processes. The exercise support component of the treatment consisted of a standardized protocol of 6,1-hour meetings with a YMCA wellness specialist, spaced across 6 months [17]. Its architecture was based on social cognitive and self-efficacy theory. These one-on-one sessions included an orientation to exercise apparatus, but most time was spent on an array of self-management/self-regulatory methods intended to foster regular exercise. Goals were identified, documented, and broken down with ongoing progress tracked graphically; and instruction in skills such as cognitive restructuring, stimulus control, and preparedness for occurrences of barriers to exercise was provided during the sessions. Specific modalities used in exercise plans (e.g., walking; stationary cycling) were based on each participant's preference. Widely used recommendations for volume of weekly exercise (150 minutes of moderate cardiovascular activity [18]) were described, but it was also suggested that any volume of exercise may be beneficial.

The nutrition component was led by a YMCA wellness specialist in a group of 12 to 15 participants. It also followed a standard protocol of educational and social-cognitive methods [19] within 6, 1-hour sessions over 3 months. Examples of program components were: 1) understanding carbohydrates, protein, fats, and calories, 2) using the U.S. Food Guide Pyramid, 3) developing a plan for snacking, and 4) a similar array of self-regulatory skills used in the exercise support component, but focused on controlled eating.

The stress management component of the treatment was embedded within the nutrition sessions in 3, 15-minute segments (along with brief practice time throughout). Methods were based on deep breathing and abbreviated progressive muscle relaxation [20]. Instruction was also given on appropriate prompts for utilization of these methods (e.g., stressful and anxious situations).

Wellness specialists administering the treatment were blind to the purposes of the investigation. Fidelity of treatment protocols was assessed by study administrators and, if 
deviations occurred, corrective measures were immediately taken. Assessments were administered in a private area at baseline and month 6 by trained exercise science professionals with advanced degrees.

\section{Data Analyses}

An intention-to-treat design was used incorporating multiple imputation [21] for the $17 \%$ of overall missing cases. Statistical significance was set at $\alpha=0.05$ (one-tailed), throughout, with the Bonferroni adjustment applied where appropriate. Within the planned multiple regression equations, to detect a small-moderate effect size of $f^{2}=0.10$ at the statistical power of 0.80 , a minimum of 112 participants was required.

Within-group changes over 6 months in BP and its hypothesized predictors associated with the treatment (resting heart rate, tension, and weight) were assessed using dependent $t$ tests, and effect sizes $\left(d=\right.$ Mean $_{\text {month } 6}-$ Mean $_{\text {baseline }} /$ $\mathrm{SD}_{\text {baseline }}$ ) were calculated where $0.2,0.5$, and 0.8 are, by convention [22], considered to be small, moderate, and large effects, respectively. Contrasts were conducted to determine if participants classified as pre-hypertensive or hypertensive differed enough on scores of the hypothesized predictors of BP change to warrant their separate analyses. The linear bivariate relationship between changes in each predictor, and BP changes, and intercorrelations within the predictors, was then calculated. Based on recent suggestions for the present type of analyses [23], actual score changes over 6 months, rather than score changes controlling for baseline values, were used. Finally, multiple regression analyses, with simultaneous entry of score changes of the predictors, were calculated so that their independent contributions to the explained variance in systolic and diastolic BP changes could be determined.

\section{RESULTS}

All participants completed at least 2 sessions of each of the 3 treatment components. Mean attendance was $72 \%$ for the exercise support, $72 \%$ for the nutrition, and $76 \%$ for the stress management components. Change scores did not significantly differ (all $P$ values $>0.35$ ) between participants with BP values consistent with pre-hypertension and hypertension. Data were, thus, aggregated for subsequent analyses.

There were significant within-group improvements in all study measures (Table 1). Effect sizes for BP change were moderate. All bivariate correlations between measures of changes in BP and changes in its predictors were in the expected direction and significant (Table 2). Intercorrelations of predictors within the planned multiple regression analyses were all low ( $r$ values $<0.20$ ), indicating their independence. Findings indicated that a significant portion of the variance in systolic and diastolic BP change was accounted for by changes in resting heart rate, tension, and weight. Only tension changes were a significant independent contributor to the explained variance in both systolic BP and diastolic $\mathrm{BP}$ reduction.

Two additional tests were conducted. In the first, changes in systolic and diastolic BP values were found to be negatively correlated with baseline values $(r \mathrm{~s}=-0.33$ and -0.47 , respectively; $P$ values $<0.001)$. In the second, age was separately stepped into both multiple regression equations as an additional predictor of BP changes. A significant change

Table 1. Changes Over 6 Months in Study Measures $(N=140)$

\begin{tabular}{|c|c|c|c|c|c|c|c|c|}
\hline & Baseline & & Month 6 & & & & & \\
\hline Measure & Mean & SD & Mean & SD & $t$ & $P$ & $95 \%$ CI & $d$ \\
\hline Systolic BP (mmHg) & 132.17 & 12.66 & 127.52 & 12.20 & -5.62 & $<0.001$ & $-6.28,-3.02$ & 0.37 \\
\hline Diastolic BP (mmHg) & 83.36 & 8.02 & 79.31 & 8.58 & -5.55 & $<0.001$ & $-5.46,-2.61$ & 0.50 \\
\hline Resting heart rate (beats/min) & 78.61 & 11.09 & 76.62 & 11.29 & -2.48 & 0.007 & $-3.58,-0.40$ & 0.18 \\
\hline Tension & 4.09 & 3.67 & 2.86 & 3.47 & -4.95 & $<0.001$ & $-1.72,-0.74$ & 0.34 \\
\hline Weight (kg) & 110.99 & 14.39 & 107.43 & 13.80 & -7.30 & $<0.001$ & $-4.53,-2.60$ & 0.25 \\
\hline
\end{tabular}

Table 2. Linear Bivariate Correlations in Study Measures $(N=140)$

\begin{tabular}{|c|c|c|c|c|c|c|c|}
\hline Measure & Mean & SD & 1 & 2 & 3 & 4 & 5 \\
\hline 1. $\Delta$ Systolic BP & -4.65 & 9.78 & - & $0.51 * * *$ & $0.18^{*}$ & $0.35 * * *$ & $0.22 * *$ \\
\hline 2. $\Delta$ Diastolic BP & -4.05 & 8.64 & $0.51 * * *$ & 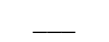 & $0.15^{*}$ & $0.20 * *$ & $0.14 *$ \\
\hline 3. $\Delta$ Resting heart rate & -1.99 & 9.51 & $0.18^{*}$ & $0.15^{*}$ & - & 0.09 & $0.16^{*}$ \\
\hline 4. $\Delta$ Tension & -1.23 & 2.94 & $0.35 * * *$ & $0.20 * *$ & 0.09 & $\underline{-}$ & $0.19 *$ \\
\hline 5. $\Delta$ Weight & -3.57 & 5.78 & $0.22 * *$ & $0.14^{*}$ & $0.16^{*}$ & $0.19 *$ & 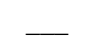 \\
\hline
\end{tabular}

The Delta symbol $(\Delta)$ denotes change from baseline to month 6 .

$* P<0.05 ; * * P<0.01 ; * * * P<0.001$. 
Table 3. Results of Multiple Regression Analyses for the Prediction of Changes in Blood Pressure $(\mathrm{N}=140)$

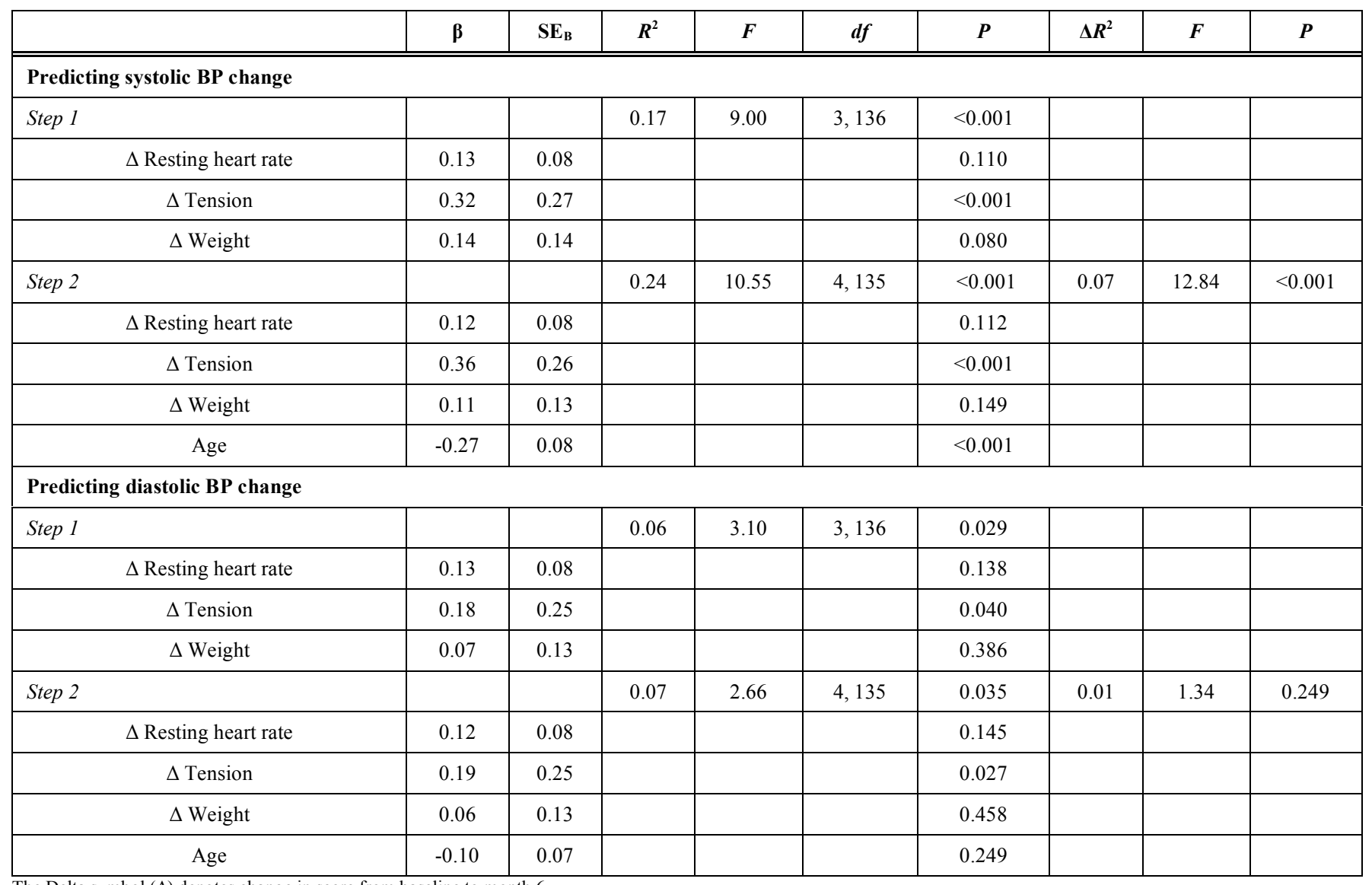

The Delta symbol $(\Delta)$ denotes change in score from baseline to month 6 .

in $R^{2}$ was found for only systolic $\mathrm{BP}$, suggesting that older age predicted greater improvement (Table $\mathbf{3}$ ).

A post hoc analysis indicated that the white $(\mathrm{n}=61)$ and black $(\mathrm{n}=72)$ participants did not significantly differ on changes in systolic BP (Mean $=-5.87, \mathrm{SD}=10.36$, and Mean $=-3.83, \mathrm{SD}=9.62$, respectively; $t_{131}=-1.17, P=$ $0.242,95 \% \mathrm{CI}=-5.46,1.39)$ or diastolic $\mathrm{BP}($ Mean $=-4.75$, $\mathrm{SD}=8.60$, and Mean $=-3.54, \mathrm{SD}=8.99$, respectively; $t_{131}=$ $-0.79, P=0.431,95 \% \mathrm{CI}=-4.25,1.82)$.

\section{DISCUSSION}

Findings supported hypothesized associations of the present community-based treatment with improvements in cardiovascular fitness, stress (tension), and weight; and their expected relationships with improved systolic and diastolic BP. Effect sizes for BP changes were comparable to more invasive and expensive behavioral treatments (e.g., individual biofeedback sessions), but generally weaker than current drug therapies [10]. Age and initial BP value were inversely associated with effect. Although a greater portion of the variance in systolic, compared to diastolic, BP was accounted for, change in tension demonstrated the most salient role for each. This finding served to fill a void in the extant research as there have been few investigations on the relative importance of behavioral predictors of lowered BP; resulting in an inability to meaningfully inform and prioritize treatment components.

Based on the present findings, the management of stress appeared to be especially important, although significant linear relationships between changes in fitness and weight, and BP changes, also indicated benefits for the inclusion of exercise and nutrition components in treatments. It was beyond the scope of this study to assess relative benefits of different stress management methods, or even the basis of the reductions in tension scores that were observed. For example, some research suggests that somatically based stress-management techniques (e.g., progressive relaxation) may be more productive than cognitively based methods (e.g., cognitive restructuring) when physical processes (e.g., BP) are addressed [24]. Also, because even moderate volumes of cardiovascular exercise, alone, have demonstrated considerable stress-reduction properties [25], occurrence of additional benefits from the inclusion of other stress-managements methods (e.g., yoga) requires careful consideration. Although this is an understudied area, it will be important for refinements in the behavioral treatment of BP.

In addition to producing useful findings for the specific subgroup if obese women sampled, this investigation was helpful for generating further questions for larger scale behavioral research where a control group is utilized to 
minimize possible confounds inherent in field-based studies. Moreover, examination of effects of pharmacological treatments combined with behavioral intervention remains needed. Limitations such as expectation effects from instructors and social support effects from fellow treatment participants may also be better dealt with in research with a control condition, although the absence of a specific focus on BP by participants was a strength. Additionally, this was a smallsample study with a specific sample of volunteers. Generalization of findings will require larger-scale testing that includes men and individuals with different degrees of overweight and other co-morbidities. Direct testing utilizing patients that were referred to treatment by their physician will enable assessment of whether their effects differ from those of volunteers (who may be especially motivated).

Although limitations were present, findings suggested that a replicable, theory-based behavioral treatment focused on exercise, improved nutrition, stress-management, and weight loss, that is applicable in community-based settings such as a YMCA, is capable of improving BP. As a result, possibilities for physicians' referral to such a treatment for addressing BP reduction appear high after considering the present findings. This may help with widespread dissemination of useful methods for a disorder with high prevalence. Ultimately, it is hoped that behavioral and medical sciences will routinely combine their efforts to maximize effects in the treatment of the increasingly common disorder of hypertension.

\section{CONFLICT OF INTEREST}

None declared.

\section{ACKNOWLEDGEMENTS}

The author acknowledges Blue Cross Blue Shield of Georgia for funding this research, and the administration of the YMCA of Metropolitan Atlanta for providing operational support.

\section{REFERENCES}

[1] Fields LE, Burt VL, Cutler JA, Huges J, Rocella EJ, Sorlie P. The burden of adult hypertension in the United States 1999 to 2000: a rising tide. Hypertension 2004; 44: 398-404.

[2] Joint national committee on prevention, detection, evaluation, and treatment of high blood pressure. The $7^{\text {th }}$ report of the joint national committee on prevention, detection, evaluation, and treatment of high blood pressure. JAMA 2003; 289: 2560-71.

[3] The Canadian consensus conference on non-pharmacological approaches to the management of high blood pressure. recommendations of the Canadian consensus conference on nonpharmacological approaches to the management of high blood pressure. CMAJ 1988; 42: 1397-409.
[4] The joint national committee on detection, evaluation, and treatment of high blood pressure. the 1988 report of the joint national committee on detection, evaluation, and treatment of high blood pressure. Arch Intern Med 1988; 148: 1023-38.

[5] Linden W, Moseley JV. The efficacy of behavioral treatments for hypertension. Appl Psychophysiol Biofeedback 2006; 31: 51-63.

[6] Church ST, Earnest CP, Skinner JS, Blair SN. Effects of different doses of physical activity on cardiorespiratory fitness among sedentary, overweight or obese postmenopausal women with elevated blood pressure. JAMA 2007; 297: 2081-91.

[7] Fox K, Borer JS, Camm AJ, et al. Resting heart rate in cardiovascular disease. J Am Coll Cardiol 2007; 50: 823-30.

[8] Fagard RH, Cornelisson VA. Effect of exercise on blood pressure control in hypertensive patients. Eur J Cardiovasc Prev Rehabil 2007; 14: 12-7.

[9] Gerin W, Pickering TG, Glynn L, et al. A historical context for behavioral models of hypertension. J Psychosom Res 2000; 48: 964-72.

[10] Linden W, Lenz JW, Con AH. Individual stress management for primary hypertension. Arch Intern Med 2001; 161: 1071-80.

[11] Neter JE, Stam BE, Kok FJ, Grobbee DE, Geleijnse JM. Influence of weight reduction on blood pressure: a meta-analysis of randomized controlled trials. Hypertension 2003; 42: 878-84.

[12] Engeli S, Sharma AM. The rennin-angiotensis system and natriuretic peptides in obesity-associated hypertension. J Mol Med 2001; 79: 21-9

[13] Laaksonen DE, Lakka H, Niskanen LK, Kaplan GA, Salonen JT, Lakka TA. Metabolic syndrome and development of diabetes mellitus. Am J Epidemiol 2002; 156: 1070-7.

[14] Bandura A. Social foundations of thought and action: a social cognitive theory. Englewood Cliffs, NJ: Prentice Hall 1986.

[15] Bandura A. Self-efficacy: the exercise of control. New York, NY: W. H. Freeman 1997.

[16] McNair DM, Heuchert JWP. Profile of Mood States technical update. North Tonawanda, NY: Multi-Health Systems 2005.

[17] Annesi JJ, Unruh JL, Marti CN, Gorjala S, Tennant G. Effects of The Coach Approach intervention on adherence to exercise in obese women: assessing mediation of social cognitive theory factors. Res Q Exerc Sport 2011; 82: 99-108.

[18] Garber CE, Blissmer B, Deschenes MR, et al. Quantity and quality of exercise for developing and maintaining cardiorespiratory, musculoskeletal, and neuromotor fitness in apparently healthy adults: guidance for prescribing exercise. Med Sci Sport Exerc 2011; 43: 1334-59.

[19] Kaiser Permanente health education services. Cultivating Health weight management kit. $7^{\text {th }}$ ed. Portland, OR: Kaiser Permanente Northwest 2008 .

[20] Bernstein DA, Given BA. Progressive relaxation: abbreviated methods. In: Woolfolk RL, Lehrer PM, Eds. Principles and practice of stress management, New York: Guilford 1984; pp. 43-69.

[21] Schafer JL. Multiple imputation: a primer. Stat Methods Med Res 1999; 8: 3-15.

[22] Cohen J. A power primer. Psychol Bull 1992; 112: 155-9.

[23] Glymour MM, Weuve J, Berkman LF, et al. When is baseline adjustment useful in analyses of change? an example with education and cognitive change. Am J Epidemiol 2005; 162: 267 78 .

[24] Lehrer PM, Woolfolk RL. Are stress reduction techniques interchangeable, or do they have specific effects? a review of the comparative empirical literature. In: Woolfolk RL, Lehrer PM, Eds. Principles and practice of stress management. New York: Guilford 1984; pp. 404-7.

[25] Annesi JJ, Whitaker AC. Relations of mood and exercise with weight loss in formerly sedentary obese women. Am J Health Behav 2008; 32: 676-83. 